\title{
In Vivo Action of 15-Lipoxygenase in Early Stages of Human Atherogenesis
}

\author{
Hartmut Kühn, Dagmar Heydeck, Isabelle Hugou, and Christina Gniwotta \\ Institute of Biochemistry, University Clinics Charité, Humboldt University, 10115 Berlin, Germany
}

\begin{abstract}
Oxidative modification of low density lipoprotein has been suggested as patho-physiologically relevant process in atherogenesis and the lipid peroxidizing enzyme 15-lipoxygenase may be involved. For experimental evidence on the in vivo action of this enzyme in the time course of plaque formation we analyzed the lipid extracts of lesional areas representing various stages of human atherogenesis for the occurrence of specific 15-lipoxygenase products. In advanced human lesions the degree of oxygenation of the lesion lipids measured as hydroxy linoleic acid/linoleic acid ratio varied between 0.2 and $3.2 \%$. Here an unspecific pattern of oxygenated lipids that did not differ from the pattern formed during copper-catalyzed LDL oxidation was detected. In both cases an enantiomer ratio (S/R-ratio) of 13-hydroxy9Z,11E-octadecadienoic acid (13-HODE) of $\sim 1: 1$ was found. In young human lesions which were obtained from the collection of the pathological determinants of atherosclerosis in youth (PDAY) program the hydroxy linoleic acid/linoleic acid ratio was much smaller (variation between 0.05 and $0.6 \%$ ), and a significant share of specific 15-lipoxygenase products was detected (S/R-ratio of 13-hydroxy linoleic acid of $54 \pm 3.1 / 46 \pm 3.1$ [mean $\pm S D]$ ]). These data suggest that the 15-lipoxygenase is enzymatically active on endogenous substrates in young human lesions and thus, may be of patho-physiological importance for early atherogenesis. In advanced human plaques the 15-lipoxygenase may be functionally silent and specific lipoxygenase products formed in earlier stages may be decomposed or superimposed by large amounts of nonenzymatic lipid peroxidation products. $(J$. Clin. Invest. 1997. 99:888-893.) Key words: eicosanoids • enantiomer separation • hydroxy fatty acids • HPLC • lipid peroxidation
\end{abstract}

\section{Introduction}

Atherosclerosis is a multifactorial disease with great socioeconomic impact $(1,2)$. Long-lasting hypercholesterolemia is of major importance, but beyond cholesterol there may be other factors of patho-physiological relevance (3). A key event in early atherogenesis is the formation of lipid loaded foam cells that accumulate in the subendothelial space of the arteries to form the fatty streaks generally accepted as early atheroscle-

Address correspondence to Dr. Hartmut Kühn, Institute of Biochemistry, University Clinics Charité, Humboldt University, Hessische Strasse 3-4, 10115 Berlin, Germany. Phone: 49-30-2093-7539; FAX: 49-30-2093-7300; E-mail: hartmut.kuehn@hpcom.rz.hu-berlin.de

Received for publication 18 September 1996 and accepted in revised form 17 December 1996.

J. Clin. Invest.

(C) The American Society for Clinical Investigation, Inc. 0021-9738/97/03/0888/06 \$2.00

Volume 99, Number 5, March 1997, 888-893 rotic lesions (4). Although the mechanism of foam cell formation in vivo is unclear it has been suggested that foam cells develop from peripheral monocytes/macrophages or from smooth muscle cells by taking up modified LDL via scavenger receptor mediated pathways $(5,6)$. Several lines of experimental evidence suggest that oxidative modification of LDL is an important process for foam cell formation $(7,8)$. There is a substantial body of experimental data on the in vitro oxidative modification of LDL (9) but the mechanism of LDL oxidation in vivo has not been investigated in detail. Immunohistochemical studies indicated the expression of a 15-lipoxygenase in human atheroma (10) and in lesions of cholesterol-fed rabbits (11), but its absence in normal vessel wall. These data and the fact that the enzyme in vitro is capable of oxidizing LDL to an atherogenic form (12-14) suggested a patho-physiological role of the enzyme in atherogenesis. However, only few experimental data are available as to the in vivo activity of this enzyme. Analyzing the lipid extracts of early atherosclerotic lesions of cholesterol fed rabbits we detected specific lipoxygenase products after a $12 \mathrm{wk}$ feeding period indicating the in vivo activity of the enzyme (15). The formation of these products appear to coincide in time with lipid deposition in the vessel wall suggesting a causal relation between 15-lipoxygenase activity and plaque formation. After $20 \mathrm{wk}$ of feeding the amount of oxidized lipids in the vessel wall was augmented but the specificity of the product pattern was significantly impaired suggesting that nonenzymatic lipid peroxidation becomes increasingly important during later stages of atherogenesis. In fact, in advanced human plaques large amounts of unspecific lipid peroxidation products were detected (16). More detailed structural elucidation of these compounds revealed a share of specific lipoxygenase products suggesting the in vivo activity of the 15-lipoxygenase in advanced human lesions (19).

The data of the above mentioned rabbit study (15) suggested that the in vivo action of the 15-lipoxygenase may be most prominent in young developing lesions. To test this hypothesis in humans we analyzed the lipid extracts of advanced human atheromas (Berlin samples) and of young human lesions obtained from the pathological determinants of atherosclerosis in youth (PDAY) ${ }^{1}$ collection (20) with respect to the specificity of oxidized lipids. The data obtained indicate that the 15-lipoxygenase expressed in young human lesions is enzymatically active and may be of patho-physiological importance in early atherogenesis.

\section{Methods}

Human atherosclerotic lesions. Specimens of human atheroma were removed during thrombaterectomy from the carotic artery. The pa-

1. Abbreviations used in this paper: CP-HPLC, chiral phase high performance liquid chromatography; 13-HODE, 13-hydroxy-9Z,11Eoctadecadienoic acid; HODE/LA ratio, hydroxyoctadecadienoic acid/ linoleic acid ration; PDAY, pathological determinants of atherosclerosis in youth; RP-HPLC, reverse phase high performance liquid chromatography. 
tients were 50-70 yr old and were selected for surgery because of clinical symptoms. In all patients a severe stenosis of the carotic artery was diagnosed by angiography. Immediately after removal the samples were shock frozen in a box with dry ice and stored at $-80^{\circ} \mathrm{C}$ until workup. Microscopic inspection indicated massive lipid deposition in all samples and signs of calcification were found in some of them. Young human atherosclerotic lesions were provided by Prof. R. Wissler (University of Chicago) from the collection of the PDAY program (20). We analyzed samples of 15-34-yr-old males and females (seven African-American males, three African-American females, seven American White males, two American White females) who died in traffic accidents or committed suicide. Samples were taken during autopsy 4-17 h after death. For the biochemical investigations the tissue samples were shock frozen and were stored in liquid nitrogen until dry ice shipment to Berlin. For histological evaluation and for immunohistochemical staining the tissue samples were imbedded in Tissue-Tec (Miles Laboratories, Inc., Elkhart, IN) and stored the same way. Microscopic inspections of freeze sections indicated a moderate lipid deposition and the presence of foamy macrophages in all samples (macrophage staining was carried out with the mouse monoclonal anti-human macrophage antibody HAM-56; Dako Corp., Carpinteria, CA). In most cases we also observed extracellular lipid deposition. Cholesterol crystals were rare and no signs of calcification could be detected. Although the cross sections have not been investigated systematically for signs of inflammation, infiltration of inflammatory cells was seen in several samples.

Preparations. Human LDL was obtained by density gradient ultra centrifugation (21). The freshly prepared LDL usually contains small amounts of oxygenated lipids (hydroxy linoleic acid/linoleic acid ratio of $\sim 0.01 \%$ suggesting that 1 out of $10^{4}$ linoleic acid residues was present as hydroxylated derivative). Enantiomer analysis indicated that these hydroxy linoleic acid isomers were racemic mixtures. We never observed a preponderance of 13S-HODE over its corresponding 13R-enantiomer. The prepared LDL was oxidized using the purified rabbit 15-lipoxygenase (enzymatic oxidation) or copper ions (nonenzymatic oxidation) as catalysts (22). The hydroperoxy lipids formed were reduced with sodium borohydride to the more stable hydroxy derivatives. After acidification the total lipids were extracted (23), hydrolyzed under alkaline conditions, and the resulting free fatty acid derivatives were analyzed by HPLC. Alkaline hydrolysis was performed under argon atmosphere in order to minimize artificial lipid peroxidation. Reverse phase-HPLC (RP-HPLC) of the hydrolyzed lipid extracts was performed at the same day to avoid lipid peroxidation during storage. For normal phase- and chiral phaseHPLC (CP-HPLC) the lipids were stored for 2 or $3 \mathrm{~d}$ under argon atmosphere at $-80^{\circ} \mathrm{C}$.

The samples of atherosclerotic tissues were thawed and areas with macroscopically visible atherosclerotic alterations were prepared. Lipid extraction was carried out with a modified Bligh/Dyer method (23) homogenizing the tissue in a mixture of methanol/chloroform/water (2:1:1, by vol). To minimize lipid peroxidation during the extraction procedure ice cold solvents were used that were freed from oxygen by bubbling extensively with argon gas.

Analytical methods. The analytical protocol for the determination of the specificity of the oxygenation products comprised three consecutive steps of HPLC; RP-HPLC followed by normal phase-HPLC and then CP-HPLC (15). Attempts to simplify the analytical procedure failed because we observed an overlapping of the hydroxy linoleic acid enantiomers with other hydroxy fatty acid isomers in CP-HPLC if they had not been separated in previous HPLC runs. In RP-HPLC the hydroxy linoleic acid/linoleic acid (HODE/LA) ratio of the hydrolyzed lipid extracts was determined. This value appears to be a suitable measure for quantification of the oxidation degree of biological lipids because it normalizes the content of the major oxidation product found in the lesions (15) to the content of the major polyenoic fatty acid. The conditions for HPLC analysis were as follows:

RP-HPLC: Nucleosil C-18 column (KS-system, $250 \times 4 \mathrm{~mm}, 5-\mu \mathrm{m}$ particle size; Macherey/Nagel, Düren, Germany); isocratic elution with methanol/water/acetic acid (82:18:0.1, by vol); flow rate, $1 \mathrm{ml} /$ min. Fractions containing oxygenated polyenoic fatty acids were collected and injected to normal phase-HPLC.

Normal phase-HPLC: Nucleosil column $(250 \times 4 \mathrm{~mm}, 5-\mu \mathrm{m}$ particle size; Macherey/Nagel); isocratic elution with $n$-hexane/2-propanol/acetic acid (100:2:0.1, by vol); flow rate, $1 \mathrm{ml} / \mathrm{min}$. Fractions containing 13-hydroxy linoleic acid (13-HODE) were collected and injected to CP-HPLC.

CP-HPLC: Chiralcel OD column $(250 \times 4 \mathrm{~mm}, 7-\mu \mathrm{m}$ particle size; Diacel Chemical Industries, distributed by J.T. Baker, Deventer, The Netherlands); isocratic elution with hexane/2-propanol/acetic acid (100:5:0.1, by vol); flow rate, $1 \mathrm{ml} / \mathrm{min}$. In all HPLC measurements the chemical structure of the hydroxy fatty acids was identified by their retention times and their ultraviolet spectrum.

Significance calculations (Student's $t$ test) were performed with the StatWorksTM1.2 program on a Macintosh Performa 450. (Apple Computers, Inc., Cupertino, CA.)

\section{Results}

15-Lipoxygenases and transition metals are capable of oxidizing human LDL to an atherogenic form $(14,22)$. There is, however, a remarkable difference in the pattern of oxidized polyenoic fatty acids formed during both reactions. For the lipoxygenase reaction 13S-hydroxy-9Z,11E-octadecadienoic acid (13S-HODE) was identified as major oxygenation product (Fig. 1). Other hydroxy fatty acid isomers including 13RHODE were minor side products. In contrast, the copper catalyzed reaction produces an unspecific product pattern of oxygenation products with a racemic mixture of $13 \mathrm{R} / \mathrm{S}-\mathrm{HODE}$ (Table I). These data suggest that the enantiomer composition (S/R-ratio) of 13-HODE may serve as suitable measure to quantify the share of specific lipoxygenase products in biological samples. In other words, a preponderance of 13S-HODE over its corresponding 13R-enantiomer in a mixture of oxidized lipids suggests the involvement of 15-lipoxygenase in the biosynthetic pathway. On the other hand, an unspecific prod-

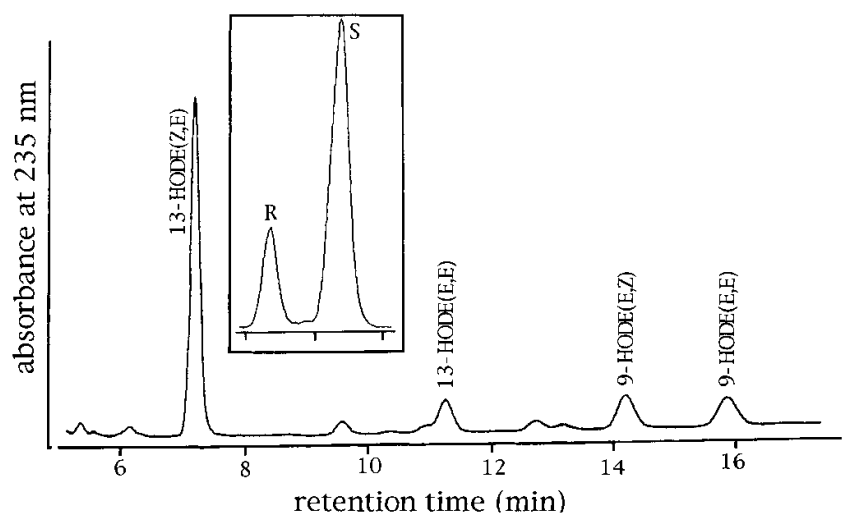

Figure 1. HPLC analysis of hydroxy fatty acids formed during 15lipoxygenase-LDL interaction. Human LDL (2 mg LDL protein/ml phosphate buffer, $\mathrm{pH}$ 7.4) was incubated with the purified rabbit reticulocyte 15-lipoxygenase (1:10 molar ratio) for $1 \mathrm{~h}$ at room temperature. The reaction was stopped by addition of sodium borohydride which reduces the hydroperoxy lipids formed to the corresponding alcohols. After acidification the lipids were extracted, hydrolyzed under alkaline conditions, and the oxygenated fatty acid derivatives were prepared by RP-HPLC. Straight phase- and CPHPLC were carried out as described in Methods. Inset: Enantiomer composition determined by chiral phase HPLC. 
Table I. Composition of Hydroxy Polyenoic Fatty Acids of In Vitro Oxidized Human LDL

\begin{tabular}{lcccc}
\hline & \multicolumn{4}{c}{ Share (\%) } \\
\cline { 2 - 5 } Catalyst & $13-\mathrm{HODE}(\mathrm{Z}, \mathrm{E})$ & 13-HODE(E,E) & $9-\mathrm{HODE}(\mathrm{Z}, \mathrm{E})$ & $9-\mathrm{HODE}(\mathrm{E}, \mathrm{E})$ \\
\hline $15-\mathrm{LOX}$ & $72 \pm 12$ & $5 \pm 4$ & $15 \pm 7$ & $6 \pm 3$ \\
$n=11$ & $(85 \pm 5: 15 \pm 5)$ & & $(51 \pm 1: 49 \pm 1)$ & \\
$\mathrm{CuSO}_{4}$ & $33 \pm 12$ & $22 \pm 8$ & $25 \pm 5$ & $20 \pm 12$ \\
$n=8$ & $(51 \pm 1: 49 \pm 1)$ & & $(50 \pm 1: 50 \pm 1)$ & \\
& & & &
\end{tabular}

Lipoxygenase-catalyzed LDL oxidation was carried out as described in the legend to Fig. 1. For copper catalyzed oxidation a 30-fold molar excess of copper over LDL was used. Analysis of the hydroxy linoleate (HODE) isomers was carried out by straight phase-HPLC. The sum of these isomers was set $100 \%$. The enantiomer composition (S/R ratio given in parentheses) was determined by chiral phase HPLC. LOXlipoxygenase.

uct pattern with a racemic mixture of 13R/S-HODE does not exclude the involvement of 15-lipoxygenase because racemization of specific products may occur (24).

In advanced human lesions the expression of 15-lipoxygenases in foamy macrophages has been reported before (10). We analyzed young human lesions obtained from the collection of the PDAY program for the expression of the 15-lipoxygenase mRNA by in situ hybridization and detected a hybridization signal in most of the freeze sections analyzed (not shown). In order to check the in vivo activity of the 15-lipoxygenase on endogenous substrates we investigated 19 PDAY samples for the enantiomer composition of 13-HODE isolated from the plaques. For comparison the lipid extracts of 17 advanced human lesions (Berlin samples) as well as the extracts of 15-lipoxygenase-treated LDL (enzymatic lipid peroxidation) and of copper-treated LDL (nonenzymatic lipid peroxidation) were analyzed. As shown in Fig. 1 during the 15-lipoxygenase catalyzed oxidation of LDL a specific product pattern with a strong preponderance of the 13S-HODE over its corresponding 13R-enantiomer was formed (Fig. 1, inset). As expected for a nonenzymatic lipid peroxidation the copper catalyzed reaction led to an unspecific product pattern as indicated by the $1: 1$ distribution of 13S- and 13R-HODE isomers (Table I). In both cases the majority of the oxidized lipids were detected in the cholesterol ester fraction (data not shown). For the lipoxygenase catalyzed reaction the degree of oxidation of the LDL lipids measured as HODE/LA ratio varied between 0.5 and $5.1 \%$ suggesting that up to 1 out of 20 linoleic acid molecules in the LDL particle was oxygenated under our experimental conditions. Increase in the incubation time and/or in the lipoxygenase concentration led to a higher HODE/LA ratio. In the lipid extracts of advanced human plaques (Berlin sample) we detected large amounts of oxygenated lipids; the HODE/LA ratio varied between 0.2 and $3.2 \%$. Here again, oxidized cholesterol esters were identified as major oxygenation products (data not shown). Because the S/R-ratio of 13-HODE isolated from these lesions was $\sim 1: 1$ (Table II) it was concluded that the vast majority of the lipid peroxidation products originate from nonenzymatic lipid peroxidation. In contrast, analysis of the oxidized lipids from young human lesions (PDAY samples) revealed a significant share $(P<0.001)$ of specific lipoxygenase products as indicated by the preponderance of $13 \mathrm{~S}$ HODE over its 13R-enantiomer (Fig. 2). In these lesions the HODE/LA ratio which varied between 0.05 and $0.6 \%$ was lower than the corresponding value for advanced atheromas. In order to exclude the possibility that the specific share of oxygenation products in the PDAY samples was formed in situ as post mortem artifact the following two control experiments was carried out. The aorta of a cholesterol-fed rabbit was removed and areas with large atherosclerotic lesions were prepared. Afterwards each lesional area was cut in half and one half was used for immediate lipid extraction. The other half was incubated for $4 \mathrm{~h}$ at $37^{\circ} \mathrm{C}$ in PBS. When both samples were taken through the experimental protocol we found that the product specificity was somewhat higher in the sample which was lipid extracted immediately after removal of the aorta (S/ R-ratio of 13-HODE of 62:48 vs. 59:41 found for the lesions incubated for $4 \mathrm{~h}$ at $37^{\circ} \mathrm{C}$ ). A similar experiment was carried out with an advanced human atheroma. Here a racemic mixture of 13-HODE (S/R ratio of 1:1) was found for both halves of the lesion. Although incubation in PBS is not equivalent to the in situ situation these data suggest that the specific share of oxygenation products found in the PDAY samples may not be formed in situ as post mortem artifact.

Table II. Enantiomer Composition of 13-Hydroxy Linoleic Acid (13-HODE) Isolated from Human Atherosclerotic Lesions and Oxidized $L D L$

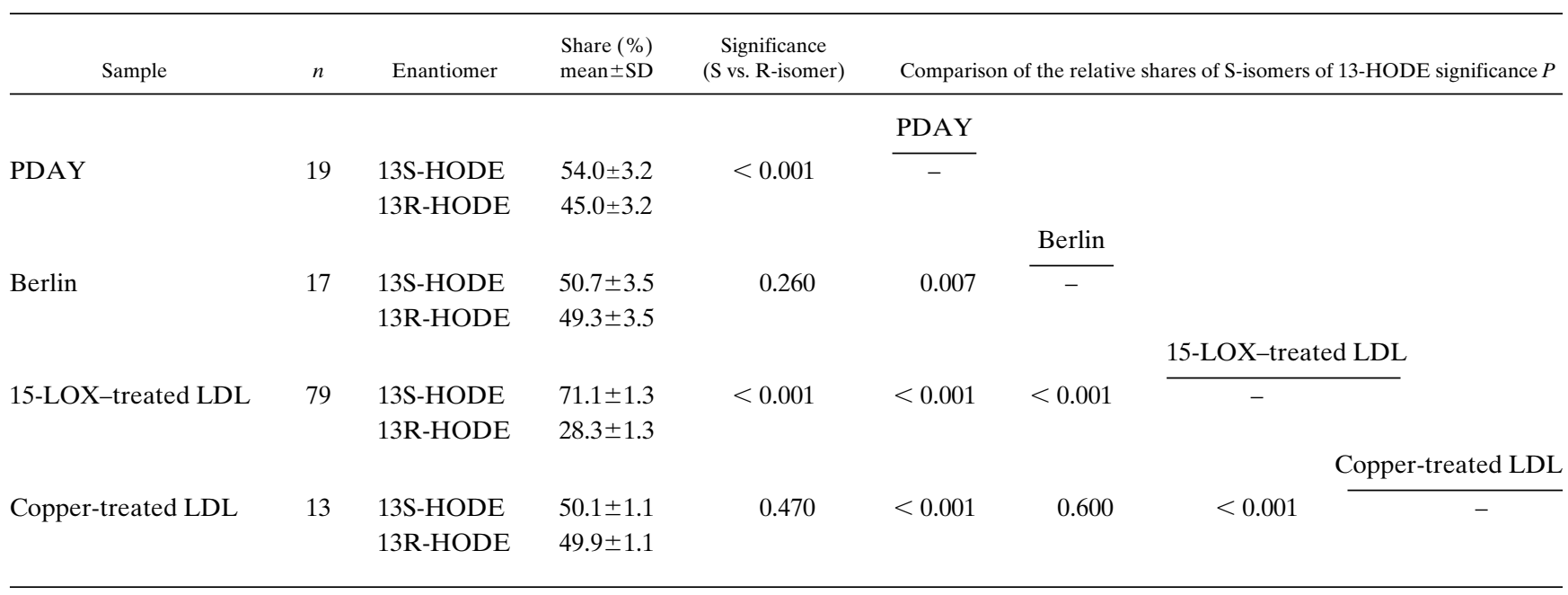




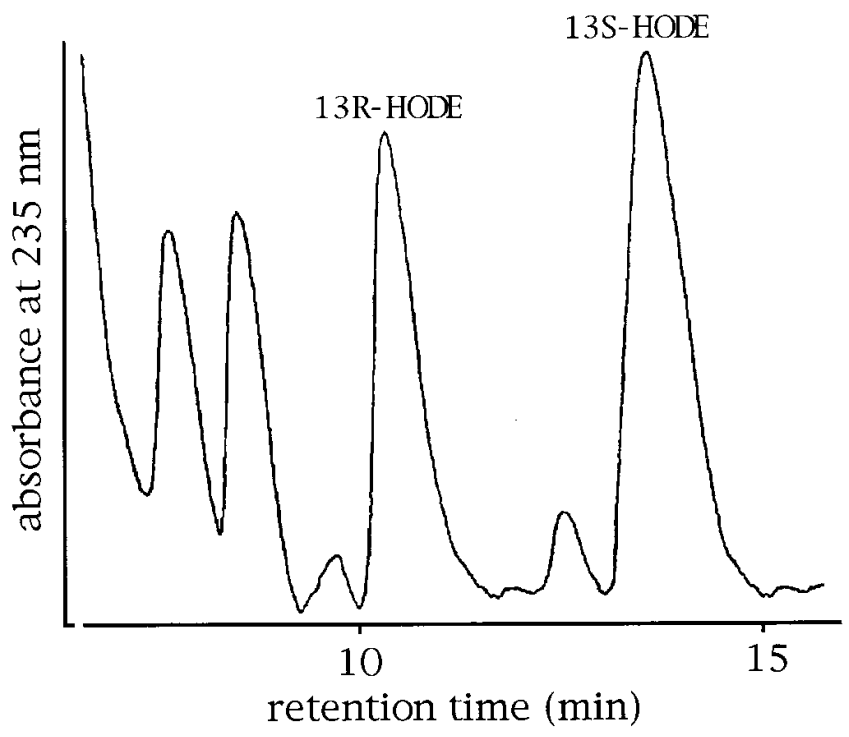

Figure 2. Enantiomer separation of 13-HODE isolated from young human atherosclerotic lesions. The lipid extract of an atherosclerotic lesion prepared from the aorta of an African-American male was hydrolyzed and 13-HODE was prepared by consecutive RP- and straight phase-HPLC as described in Methods. After removal of the solvent 13-HODE was reconstituted in hexane/2-propanol/acetic acid (100:5:0.5, by vol) and the enantiomer composition was determined by CP-HPLC (Methods). The ultraviolet spectra of both 13-HODE enantiomers were characterized by a conjugated diene chromophore (not shown). In this case, a S/R-ration of 59/41 was determined.

The quantification of the enantiomer analysis of the 13HODE prepared from oxidized LDL and from the human lesions as well as the statistical evaluation of the data are summarized in Table II. As expected for nonenzymatic lipid peroxidation the oxidized lipids of copper-treated LDL and of advanced human lesions were racemic mixtures; there was no significant difference between the relative shares of the 13Sand 13R-HODE ( $P=0.47$ and 0.26 , respectively). In contrast, the oxidized lipids from young human lesions and from 15lipoxygenase treated LDL were characterized by a significant difference $(P<0.001)$ between the relative amounts of $13 \mathrm{~S}$ and 13R-HODE. Comparing the relative amounts of $13 \mathrm{~S}-$ HODE found in the various samples (Table II, left) the following major conclusions may be drawn:

(a) There was no significant difference in the specificity of the oxidized lipids isolated from advanced human lesions and from copper treated LDL $(50.7 \pm 3.5 \%$ vs. $50.1 \pm 1.1 \%$ respectively; $P=0.530$ ) suggesting that the vast majority of the oxidized lipids in these lesions may originate from nonenzymatic lipid peroxidation. (b) The specificity of the oxidized lipids in young human lesions was significantly higher than that of advanced plaques as indicated by the higher share of 13S-HODE (54.0 \pm 3.2 vs. $50.7 \pm 3.5$ respectively; $P=0.007)$. These data suggest that the 15-lipoxygenase may contribute to lipid peroxidation mainly in young human lesions. $(c)$ The product specificity of 15-lipoxygenase-treated LDL was higher than that of the PDAY samples (share of $13 \mathrm{~S}-\mathrm{HODE}$ of $71.7 \pm 1.1 \%$ in lipoxygenase treated LDL vs. 54.0 \pm 3.2 in the PDAY samples, $P<0.001$ ) suggesting that the 15-lipoxygenase may not be the only source of oxidized lipids in young human lesions.

\section{Discussion}

Although oxidative modification of LDL appears to be an important process in atherogenesis the role of 15-lipoxygenase remains a matter of discussion. The data presented here indicate that specific oxygenation products can be detected in young developing human atherosclerotic lesions. These products may be formed by the in vivo action of the 15-lipoxygenase which is expressed in foamy macrophages. In more advanced human lesions we failed to detect specifically oxygenated lipids. It should be stressed at this point that a specific share of lipid peroxidation products does not prove the in vivo action of a 15-lipoxygenase. In complex biological environments such as a developing plaque other enantiospecific metabolic pathways may occur. Recently, it has been reported that myoglobin catalyzes the stereospecific oxygenation of linoleic acid in the presence of hydrogen peroxide. As major reaction product 9-HPODE with an S/R-ratio of $\sim 8: 2$ was identified (25). Remarkably, no 13-H(P)ODE was found. Because we evaluated the S/R-ratio of 13-HODE in this study myoglobin catalysis may not be relevant. As alternative explanation for an S/R-ratio higher than 1 an enantiospecific metabolization of 13R-HODE from a racemic mixture may be discussed. However, when we incubated a homogenate of rabbit atherosclerotic lesions with a racemic mixture of 13-HODE no evidence for such a stereoselective secondary metabolization was obtained.

In addition to the results presented here several lines of experimental evidence obtained in different laboratories suggest a patho-physiological role of the 15-lipoxygenase in atherogenesis. (a) The 15-lipoxygenase is expressed in foamy macrophages $(10,11)$ as well as in certain types of smooth muscle cells (26) of atherosclerotic lesions. Because the enzyme is not expressed in the normal human arterial wall (10) nor in macrophage precursor cells (27) there must be a specific induction of the 15-lipoxygenase during atherogenesis. $(b)$ The in vivo activity of the 15-lipoxygenase appears to coincide in time with the onset of lipid deposition in the vessel wall in cholesterol fed rabbits (15). (c) Somatic gene transfer of the 15-lipoxygenase to a rabbit iliac artery led to the appearance of oxidized LDL epitopes in the transfected areas (28). In contrast, in the contralateral iliac artery that was mock transfected such epitopes were not detected. (d) A 15-lipoxygenase inhibitor which lacks antioxidative properties appears to inhibit the development of atherosclerotic lesions in cholesterol fed rabbits (29). Although these data suggest a role of the enzyme in atherogenesis the molecular mechanism of its action is unclear.

Since the 15-lipoxygenase in vitro is capable of oxidizing LDL to an atherogenic form $(14,22)$ and because fibroblasts transfected with the 15-lipoxygenase cDNA exhibit a higher LDL oxidizing capability than mock-transfected cells (30) one may conclude that the enzyme in vivo may be involved in LDL oxidation. There are several hypothesis to explain how an intracellular lipoxygenase can oxidize extracellular $\operatorname{LDL}(30,31)$ but additional experimentation is needed to verify them. On the other hand, initiation of extracellular LDL oxidation may not be the only and, most probably, not even the major action of the enzyme during atherogenesis. It might be possible that the 15-lipoxygenase is induced in foam cells as a part of a lipid metabolizing cascade designed to prevent intracellular lipid deposition (32). Thus, for further mechanistic studies even an antiatherogenic action of the 15-lipoxygenase may be considered $(32,33)$. In fact, transgenic rabbits which overexpress the 
15-lipoxygenase specifically in monocyte/macrophages (34) appear to be protected from the development of atherosclerotic lesions during cholesterol feeding (32).

The statistical evaluation of the product specificity of the oxygenated lipids prepared from young human lesions indicated a rather small departure from randomness which was, however, highly significant. Previous studies on the time course of lesion development in cholesterol fed rabbits (15) revealed a much more specific product pattern in early-stage lesions which rendered more unspecific at later time points of the feeding period. Because the PDAY samples do not represent very early lesions (such lesions may be obtained from animal atherosclerosis models only) the departure from randomness was not expected to be as obvious as in the rabbit study. During the course of lesion development secondary reactions may impair the specificity of the oxygenation products. It has been shown that specific hydroperoxy lipids formed by the 15lipoxygenase may isomerize if they are not rapidly reduced. Moreover, specific hydroperoxy lipids formed by the lipoxygenase may initiate nonenzymatic lipid peroxidation that also renders the product pattern more unspecific. It should be stressed that even if the initiation of LDL oxidation in vivo were totally dependent upon 15-lipoxygenase nonenzymatic propagation reactions would quickly wipe out any major excess of the S-isomer, even if the chain length of propagation would be relatively short. Taking into account these mechanistic considerations one cannot expect a much more prominent departure from randomness if one examines human lesions.

Recently Folcik et al. (19) reported that the pattern of hydroxylated fatty acids in advanced human lesions showed a significant departure from randomness suggesting the in vivo action of a lipoxygenase. As controls the S/R-ratio of 13-HODE formed during copper-catalyzed LDL oxidation was determined. The data obtained in this study for advanced human lesions do not confirm these results. Although the reasons for this apparent discrepancy are unclear, analytical problems may be involved. With the "Pirkle-type" chiral column used in the Folcik study (19) there is a partial overlap of 13R- and 13SHODE. Such overlaps may sometimes cause problems when the chromatograms are quantified by peak areas. On the cyclodextrin-based chiral columns (35) used in this study the 13HODE enantiomers are baseline resolved. Surprisingly, we detected in most extracts of the PDAY and of the Berlin samples an unknown compound containing a conjugated diene chromophore which migrated between 13S- and 13R-HODE (Fig. 2). If this compound would coelute with 13S-HODE on the "Pirkle-type" column the amount of this enantiomer would be overestimated.

As alternative explanation for the apparent discrepancy of the earlier studies a variable activity status of the enzyme in the lesion may be discussed. A lack of specific oxygenation products suggests that the lipoxygenase may be inactive on endogenous substrates. It might be possible that the enzyme is activated during the surgical plaque removal. Thus, differences in the surgical procedure may lead to a different product pattern. On the other hand, the endogenous lipoxygenase activity and, thus, the amount of the specific lipoxygenase products may depend on the activity status of the plaque. In resting lesions the 15-lipoxygenase may be inactive and thus specific oxygenation products may be absent. In active lesions with areas of growth and cell proliferation the 15-lipoxygenase may be activated and, thus, specific lipoxygenase may be formed. It is well known that intracellular 15-lipoxygenases require activation for their catalytical action (36). Recently, we observed that the membranes of smooth muscle cells transfected with the 15-lipoxygenase only contain specific lipoxygenase products if the cells proliferate (unpublished data). The membranes of nondividing cells do not contain these products. Although these observations suggest a correlation of intracellular lipoxygenase activity and cell proliferation more work is needed to provide experimental evidence for such a correlation during lesion development in vivo.

\section{Acknowledgments}

The authors are indebted to Prof. R. Wissler and his colleagues from the PDAY project for providing samples of young human atherosclerotic lesions.

This work was supported by the European Community (PL 931790) and by Deutsche Forschungsgemeinschaft (Ku 961/1-2).

\section{References}

1. Ross, R. 1993. The pathogenesis of atherosclerosis: a perspective for the 1990s. Nature (Lond.). 362:801-809.

2. Steinberg, D., and J.L. Witztum. 1990. Lipoproteins and atherogenesis. JAMA (J. Am. Med. Assoc.). 264:3047-3052.

3. Steinberg, D., S. Parthasarathy, T.E., Carew, J.C. Khoo, and J.L. Witztum. 1989. Beyond cholesterol. Modification of low density lipoprotein that increase its atherogenicity. N. Engl. J. Med. 320:915-924.

4. Takano, T., and C. Mineo. 1990. Atherosclerosis and molecular pathology: Mechanism of cholesterol ester accumulation in foam cells and extracellular space of atherosclerotic lesions. J. Pharmacobiodyn. 13:385-413.

5. Aqel, N.M., R.Y. Ball, H. Waldman, and M.J. Mitchinson. 1984. Monocyte origin of foam cells in human atherosclerotic plaques. Atherosclerosis 53: $265-271$.

6. Amanuma, K., T. Kanaseki, S. Ikeuchi, S. Ohkuma, and T. Takano. 1986 Studies on fine structure and location of lipids in quick-freeze replicas of atherosclerotic aorta in WHHL rabbits. Virchows Arch. 410:231-238.

7. Ylä-Herttuala, S., W. Palinski, M.E. Rosenfeld, S. Parthasarathy, T.E. Carew, S. Butler, J.L. Wiztum, and D. Steinberg. 1989. Evidence for the presence of oxidatively modified low density lipoprotein in atherosclerotic lesions of rabbit and man. J. Clin. Invest. 84:1086-1059.

8. Holvoet, P., and C. Collen. 1994. Oxidized lipoproteins in atherosclerosis and thrombosis. FASEB J. 8:1284-1297.

9. Esterbauer, H., and P. Ramos. 1995. Chemistry and Pathophysiology of oxidation of LDL. Rev. Physiol. Biochem. Pharmacol. 127:31-34.

10. Ylä-Herttuala, S., M.E. Rosenfeld, S. Parthasarathy, C.K. Glass, E. Sigal, T. Sarkioia, J.T. Witztum, and D. Steinberg. 1991. Gene expression in macrophage rich human atherosclerotic lesions. J. Clin. Invest. 87:1146-1152.

11. Ylä-Herttuala, S., M.E. Rosenfeld, S. Parthasarathy, C.K. Glass, E. Sigal, J.T., Witztum, and D. Steinberg. 1990. Colocalization of 15-lipoxygenase mRNA and protein with epitopes of oxidized low density lipoprotein in macrophage-rich areas of atherosclerotic lesions. Proc. Natl. Acad. Sci. USA. 87: 6959-6936.

12. Sparrow, P.C, S. Parthasarathy, and D. Steinberg. 1988. Enzymatic modification of low density lipoprotein by purified lipoxygenase plus phospholipase $\mathrm{A}_{2}$ mimics cell-mediated oxidative modification. J. Lipid Res. 29:745-753.

13. Cathcart, M.K., A.K. McNally, G.M. Chisolm. 1991. Lipoxygenase mediated transformation of human low density lipoprotein to an oxidized and cytotoxic complex. J. Lipid Res. 32:63-70.

14. Belkner, J., R. Wiesner, J. Rathman, J. Barnett, E. Sigal, and H. Kühn. 1993. Oxygenation of lipoproteins by mammalian lipoxygenases. Eur. J. Biochem. 213:251-261.

15. Kühn, H., J. Belkner, S. Zaiss, T. Fährenklemper, and S. Wohlfeil. 1994 Involvement of 15-lipoxygenase in early stages of atherogenesis. J. Exp. Med. 179:1903-1911.

16. Harland, W.A., J.D. Gilbert, G. Steel, and J.W. Brooks. 1971. Lipids of human atheroma. Atherosclerosis. 13:239-246.

17. Kühn, H., J. Belkner, R. Wiesner, T. Schewe, V.Z. Lankin, and A.K. Tikhaze. 1992. Structure elucidation of oxygenated lipids in human atherosclerotic lesions. Eicosanoids. 5:17-22.

18. Harland, W.A., J.D. Gilbert, and C.J. Brooks. 1973. Lipids of human atheroma. VIII. Oxidised derivatives of cholesteryl linoleate. Biochim. Biophys. Acta. 316:378-385.

19. Folcik, V.A., R.A. Nivar-Aristy, L.P. Krajewski, and M.K. Cathcardt. 1995. Lipoxygenase contributes to the oxidation of lipids in human atherosclerotic plaques. J. Clin. Invest. 96:504-510. 
20. Wissler, R. 1994. New insights into the pathogenesis of atherosclerosis as revealed by PDAY. Atherosclerosis. 108:S3-S20.

21. Redgrave, T.G., D.C.K. Roberts, and C.E. West. 1975. Separation of plasma lipoproteins by density-gradient ultracentrifugation. Anal. Biochem. 65: $42-49$.

22. Lass, A., J. Belkner, H. Esterbauer, and H. Kühn. 1996. Lipoxygenase treatment renders low density lipoprotein susceptible to copper catalyzed oxidation. Biochem. J. 314:577-585.

23. Bligh, E.G., and W.J. Dyer. 1959. A rapid method of total lipid extraction and purification. Can. J. Biochem. Biophysiol. 37:911-917.

24. Schnurr, K., J. Belkner, F. Ursini, T. Schewe, and H. Kühn. 1996. The selenoenzyme phospholipid hydroperoxide glutathione peroxidase controls the activity of the 15-lipoxygenase with complex substrates and preserves the specificity of the oxygenation products. J. Biol. Chem. 271:4653-4658.

25. Rao, S.I., A. Wilks, M. Hamberg, and P.R. Ortiz de Montellano. 1994. The lipoxygenase activity of myoglobin. J. Biol. Chem. 269:7210-7216.

26. Hugou, I., P. Blin, J. Henri, D. Daret, and J. Larrue. 1995. 15-lipoxygenase expression in smooth muscle cells from atherosclerotic rabbit aorta. Atherosclerosis. 113:189-195.

27. Conrad, D.J., H. Kühn, M. Mulkins, E. Highland, and E. Sigal. 1992. Specific inflammatory cytokines regulate the expression of human monocyte 15-lipoxygenase. Proc. Natl. Acad. Sci. USA. 89:217-221.

28. Ylä-Herttuala, S., J. Luoma, H. Viita, T. Hiltunen, and T. Nikkari T. 1995. Transfer of 15-lipoxygenase gene into rabbit iliac arteries results in the appearence of oxidation-specific lipid protein adducts characteristic of oxidized low density lipoprotein J. Clin. Invest. 95:2692-2698.

29. Cornicelli, J.A., S. Sendobry, T. Bocan, K. Welch, B. Tait, B.K. Trivedi, N. Colbry, and S. Feinmark. 1995. A novel 15-lipoxygenase inhibitor lacking antioxidative properties prevents atherosclerosis in cholesterol fed rabbits. XII International Symposium on Drugs Effecting Lipid Metabolism. Nov. 7-10. 54 (Abstr.).

30. Benz, D.J., M. Mol, M. Ezaki, N. Mori-Ito, I. Zelan, A. Miyanohara, T. Friedman, S. Parthasarathy, D. Steinberg, and J.L. Witztum. 1995. Enhanced levels of lipopreoxides in low density lipoprotein incubated with murine fibroblast expressing high levels of human 15-lipoxygenase. J. Biol. Chem. 270:51915197.

31. Kühn, H., L. Chan. 1997. The role of 15-lipoxygenase in atherogenesis. Pro- and/or anti-atherogenic action. Curr. Opin. Lipidol. In press.

32. Shen, J., E. Herderick, F. Cornhill, E. Zsigmond, H.S. Kim, H. Kühn, N.V. Guevara, L. Chan. 1996 Macrophage-mediated 15-lipoxygernase expression protects against atherosclerosis development. J. Clin. Invest. 98:2201-2208.

33. Simon, T.C., A.N. Makheja, and J.M. Bailey. 1989. The induced lipoxygenase in atherosclerotic aorta converts linoleic acid to the platelet chemorepellant factor 13-HODE. Thromb. Res. 55:171-178.

34. Shen, J., H. Kühn, A. Petho-Schramm, and L. Chan. 1995. Transgenic rabbits with the integrated human 15-lipoxygenase gene driven by a lysozyme promotor: macrophage-specific expression and variable positional specificity of the transgenic enzyme. FASEB J. 9:1623-1631.

35. Wiesner, R., and H. Kühn. 1993. High performance liquid chromatography of oxygenated polyenoic fatty acids including enantiomer separation. In CRC-Handbook of Chromatography: Analysis of Lipids. K.D. Mukherjee and N. Weber, editors. CRC Press, Inc., Boca Raton, FL. 89-100.

36. Kühn, H. 1997. Biosynthesis, metabolization and biological importance of the primary 15-lipoxygenase metabolites 15 -hydro(pero)xy-5Z,8Z,11Z,13Eeicosatetraenoic acid and 13-hydro(pero)xy-9Z,11E-octadecadienoic acid. Prog. Lipid. Res. 35:203-206. 